\title{
Coulomb Corrections to Elastic Electron-Proton Scattering and the Proton Charge Radius
}

\author{
R. Rosenfelder 周 \\ Paul Scherrer Institut, CH-5232 Villigen PSI, Switzerland
}

\begin{abstract}
Recent values of the proton charge radius derived from the Lamb shift in electronic hydrogen tend to be larger than those from electron scattering. Therefore low-momentum-transfer scattering data from different groups and laboratories have been reanalyzed with Coulomb and recoil corrections included. This was done by calculating at each scattering angle and scattering energy the corresponding correction obtained from a partial wave program which includes recoil approximately. Corrections due to magnetic scattering were also made before the rms-radius was determined by a fit which allows free normalization for each experiment. It is shown that an analysis of electron scattering data including Coulomb corrections lowers the $\chi^{2}$ of the fit and increases the proton radius by about $(0.008-0.013) \mathrm{fm}$ depending on the fit strategy. A value of $r_{p}=0.880(15) \mathrm{fm}$ is obtained which is in good agreement with the one extracted from Lamb shift measurements.
\end{abstract}

${ }^{*}$ E-mail address: rosenfelder@psi.ch 
1. Traditionally elastic electron scattering data from the proton have been analyzed in the first Born (one-photon-exchange) approximation since $Z \alpha \simeq 1 / 137$ was supposed to be small enough. In this way a root-mean-square charge radius of

$$
<r^{2}>_{\mathrm{e}-\text { scatt }}^{1 / 2}=0.862(12) \mathrm{fm}
$$

has been deduced from elastic electron-proton scattering experiments at Mainz [1] 20 years ago. This value superseded the nearly canonical value of 0.805(11) fm from Stanford [2] which was based on an (invalid) $Q^{2} \rightarrow 0$ extrapolation of the dipole form factor fitted to data at higher momentum transfer $\square$. In the last few years atomic Lamb shift measurements in hydrogen have become increasingly more accurate and are now very sensitive to the proton size [5]. Assuming the validity of bound state QED calculations a value for the proton charge radius can be deduced from them; the most recent theoretical evaluation [6] leads to

$$
<r^{2}>_{\text {Lamb shift }}^{1 / 2}=0.883(14) \mathrm{fm}
$$

which is slighthly higher than the value (11). A possible reason of this discrepancy may be the distortion of the scattering waves in the electrostatic field of the proton. Indeed, Coulomb corrections in elastic electron scattering have been shown recently to increase the rms charge radius of the $Z=1, N=1$ hydrogen isotope, the deuteron by $0.012-0.015 \mathrm{fm}$ [7], [8]. Therefore it is of some interest to investigate whether Coulomb corrections also affect the determination of the proton radius. Eventually a muonic hydrogen experiment which has started at PSI [9] will yield this fundamental hadronic quantity with a tenfold higher accuracy than given in (1) or in (2). Even then it will be useful to see whether the value from electron scattering is consistent with the precise value.

2. In the one-photon-exchange approximation the elastic electron-proton scattering cross section in the lab frame is given by

$$
\left.\frac{d \sigma}{d \Omega}\right|_{1-\text { photon }}=\sigma_{\text {Mott }} f_{\text {recoil }}\left[\frac{G_{E}^{2}\left(Q^{2}\right)+\tau G_{M}^{2}\left(Q^{2}\right)}{1+\tau}+2 \tau G_{M}^{2}\left(Q^{2}\right) \tan ^{2}\left(\frac{\Theta}{2}\right)\right]
$$

where $\tau=Q^{2} / 4 M_{p}, Q^{2}=4 E_{i} E_{f} \sin ^{2}(\Theta / 2) . \quad E_{i}, E_{f}, \Theta$ are the initial, final electron energy and the scattering angle, respectively. The recoil factor is given by $f_{\text {recoil }}=\left[1+2 E_{i} \sin ^{2}(\Theta / 2) / M_{p}\right]^{-1}$ and $G_{E / M}\left(Q^{2}\right)$ denote the electric and magnetic (Sachs) form factors. The former has the low $Q^{2}$ expansion

$$
G_{E}\left(Q^{2}\right)=1-\frac{1}{6}\left\langle r^{2}\right\rangle Q^{2}+\frac{1}{120}\left\langle r^{4}\right\rangle Q^{4}+\ldots
$$

from which the root-mean-square charge radius $r_{p} \equiv\left\langle r^{2}\right\rangle^{1 / 2}$ of the proton can be determined. At low momentum transfer the magnetic form factor is only a small correction and and a common approximation is to assume $G_{M}\left(Q^{2}\right)=\mu_{p} G_{E}\left(Q^{2}\right)$ where $\mu_{p}=2.793$ is the magnetic moment of the proton. Therefore the full cross section may be written as

$$
\frac{d \sigma}{d \Omega}=\sigma_{\text {Mott }} f_{\text {recoil }} \frac{G_{E}^{2}\left(Q^{2}\right)}{1+\tau}\left(1+\Delta_{\text {mag }}\right)\left(1+\Delta_{c}\right)
$$

\footnotetext{
${ }^{1}$ Although the deviation of the data at low momentum transfer from the standard dipole parametrization is clearly visible and unambigous (see e.g. Fig. 4 in ref. [3]) the Stanford value is still cited as an alternative in the (atomic and high energy physics) literature. However, the precise absolute e-p cross sections measured by the Walther group at Mainz have been confirmed subsequently in other measurements and laboratories (most lately at Saclay, see Fig. 2 in ref. [4] ) so that there is no convincing reason for doing so.
} 
where

$$
\Delta_{\mathrm{mag}}=\frac{G_{M}^{2}\left(Q^{2}\right)}{G_{E}^{2}\left(Q^{2}\right)} \tau\left[1+2(1+\tau) \tan ^{2}\left(\frac{\Theta}{2}\right)\right] .
$$

Here it is assumed that the Coulomb corrections affect the magnetic part in the same way as the electric part; in any case eq. (5) is correct if both corrections are small.

To evaluate the Coulomb correction $\Delta_{c}$ one may either calculate the second Born approximation or use a standard partial wave program which solves the Dirac equation in the electrostatic potential $V_{c}$ of the proton. Here I adopt the second approach which is numerically easier since it does not require principal value integrations. The drawback is that magnetic scattering is not included and that it is difficult to implement recoil corrections properly which naturally are more important for electron scattering from the proton than from heavier targets. Following ref. [10] I transform to the center-of-mass system but neglect dynamical recoil corrections, i.e. the additional interaction potential $\Delta V$ which is of order $V_{c}^{2} / M_{p}$. This is a standard procedure since the work of Foldy et al. 111 and has been shown to produce Coulomb corrections at low momentum transfer which are very close to the one from evaluating the real part of the second-order Born amplitude [8]. Of course, the Coulomb corrections require knowledge of $V_{c}(r)$, i.e. of the whole charge distribution and not just of its second moment. From eq. (5) one can read off

$$
\rho_{c}(r)=\int \frac{d^{3} q}{(2 \pi)^{3}} \frac{G_{E}\left(\mathbf{q}^{2}\right)}{\sqrt{1+\mathbf{q}^{2} /\left(4 M_{p}^{2}\right)}} e^{i \mathbf{q} \cdot \mathbf{r}} ; \quad V_{c}(r)=\int d^{3} x \frac{\rho_{c}(x)}{|\mathbf{r}-\mathbf{x}|}
$$

if the leading term is taken as square of an elastic form factor which itself is the Fourier transform of a charge distribution. Since (in the Breit frame) the Sachs form factors can indeed be interpreted as arising from appropriate distributions, Eq. (7) just describes the folding of this distribution with the one from the Darwin-Foldy term. For $G_{E}\left(\mathbf{q}^{2}\right)$ I have used the parametrization given in eq. (9) and Table 3 of ref. [1] which describes the form factor data over a wide range of $Q^{2}$ and which corresponds to a sum of Yukawa charge distributions. The folding was performed by a Fourier-Bessel expansion of the distribution with a cut-off radius of $5 \mathrm{fm}$ and up to 300 terms. The Coulomb correction for each scattering angle and energy is then given by

$$
\Delta_{c}^{(i)}=\frac{\sigma_{\text {partial wave }}\left(E_{i}, \Theta_{i}\right)}{\sigma_{\text {charge, Born }}\left(E_{i}, \Theta_{i}\right)}-1
$$

where $\sigma_{\text {charge, Born }}$ is the cross section in Born approximation due to the charge distribution $\rho_{c}(r)$. For small scattering angles the Coulomb corrections are all positive leading to an increase of the rms radius [8].

3. I have (re)analyzed the old e-p cross section data below $Q^{2}<1 \mathrm{fm}^{-2}$ from Mainz and Saskatoon. More recent experiments are either at higher $Q^{2}$ [12] or measure form factor ratios by polarization transfer [13]. The Mainz data include published cross sections from the Walther group [1, 14, 15, 3] as well as unpublished data contained in the $\mathrm{PhD}$ thesis by W. Reuter 16. The Saskatoon experiment 17] detected the struck proton but the data can be easily converted to electron angles. In the following these experimental data sets will be denoted as S1, S2, B1, B2, R and M, respectively. For ease of comparison and further reference the cross sections, their quoted errors, and the corrections $\Delta_{\text {mag }}, \Delta_{c}$ are collected in Table 1 as well as the resulting values of the electric form factor with errors. Note that in the latest two experiments by Simon et al. (abbreviated S1, S2) the systematic errors were added 
linearly to the random errors whereas the other experiments do not specify how their systematic errors are treated. In view of that their total cross section errors may be underestimated. The correction due to magnetic scattering has been obtained with the parametrizations for $G_{M / E}$ as given in ref. [1]; its relative error was estimated to be $3 \cdot 10^{-3}$ by comparing with the ansatz $G_{M}=G_{E}$. From Table 1 one sees that the Coulomb corrections are small but positive, ranging from $0.4-0.9 \%$ which is roughly the size of the statistical or systematic errors in the experiments of Simon et al. By comparing with the result of a phase shift calculation, which uses an exponential distribution with the same rms-radius, an error of at most $2 \cdot 10^{-4}$ can be ascribed to the Coulomb corrections listed in Table 1. Qualitatively one therefore expects that the analysis leads to an upward shift of the proton radius of roughly the order of the error which was quoted in previous analyses.

This is indeed born out by a fit of the electric form factor at low momentum transfers to the expression

$$
G_{E}^{\text {ansatz }}\left(Q^{2}\right)=a_{0}+a_{1} Q^{2}+a_{2} Q^{4} .
$$

It has been recognized very early that the normalization errors $\delta_{\text {norm }}$ in absolute cross section measurements require a free normalization factor $a_{0}$ in eq. (9). Wong [18] has further emphasized that the normalization errors have to be part of the $\chi^{2}$-function

$$
\chi^{2}=\sum_{i=1}^{n}\left(\frac{G_{E}^{(i) \exp }-G_{E}^{(i) \text { ansatz }}}{\delta G_{E}^{(i) \exp }}\right)^{2}+\sum_{j}\left(\frac{1-a_{0}^{(j)}}{\frac{1}{2} \delta_{\text {norm }}^{(j)}}\right)^{2}
$$

where $i$ runs over the $n$ experimental points and $j$ over the different experiments. The factor $1 / 2$ in the denominator of the last term in eq. (10) enters because the form factor and not the cross section is fitted. According to Wong's analysis of the Mainz data, "the result would have been

$$
r_{p}^{\text {Wong }}=0.877(24) \mathrm{fm}
$$

with $a_{0}=1.0028(22)$ ". Since only low $Q^{2}$-data are analyzed the coefficient $a_{2}$ is not determined well by the fit. A value $a_{2} / a_{0}=0.011 \pm 0.004 \mathrm{fm}^{4}$ has been determined in ref. [19]. However, since the S1-parametrization of $G_{E}$ is a very good representation of the available e-p data 9 I rather adopt its value of $a_{2}=0.0141 \mathrm{fm}^{4}$ and use in most of the fits

$$
\frac{a_{2}}{a_{0}}=(0.014 \pm 0.004) \mathrm{fm}^{4} .
$$

Note that the error assumed above corresponds to a $7 \%$ error in the moment $<r^{4}>^{1 / 4}$ which probably is a generous estimate. This error (muliplied by $Q^{4}$ ) is now added linearly to the errors of $G_{E}$ listed in Table 1.

4. The results of fitting eq. (9) to the data collected in Table 1 are given in Table 2, both without (case A) and with (case B) Coulomb corrections. Several fits have been made differing in additional constraints for the size of the corrections. One constraint already used in Table 1 is a low momentum tranfer so that the recoil corrections do not become too large; another one is the dominance of charge scattering for which the Coulomb corrections are most reliable. In nearly all cases the magnetic contributions were therefore restricted to be less than $10 \%$. Fit 3 gives the results when this is relaxed

\footnotetext{
${ }^{2}$ Platchkov et al. [4] have found that their absolute proton cross sections measured in the momentum transfer region between 1 and $16 \mathrm{fm}^{-2}$ "agree within $1 \%$ with the four-pole proton form factor parametrization of Simon et al."
} 
to $\Delta_{\text {mag }}<15 \%$. The result of fit $1 \mathrm{~A}$ is very close to the original Mainz proton radius (西) although it uses an unrealistic small value of $a_{2}$. In fit 4 only the data of the Walther group are analyzed; without Coulomb corrections this indeed gives a value of the proton radius which is in agreement with Wong's one in eq. (11). However, for unknown reasons I do not observe that "uncertainties in the normalization factor ... have significantly increased the error in the deduced radius" $"$. Fit 5 omits the more indirect Sakatoon data (abbreviated by $\mathrm{M}$ ) which give the largest individual contribution to the $\chi^{2}$. Finally in fit 6 the errors in data sets R, B1, B2, M were artificially enlarged by a factor of 2 to take into account systematic errors which are not included in the original data.

In all cases one observes that the inclusion of Coulomb corrections improves the $\chi^{2}$ of the fit and increases the deduced proton radius by

$$
\Delta r_{p}=(0.008-0.013) \mathrm{fm}
$$

depending on the different fit strategies adopted. For the fits with $\Delta_{\text {mag }}<0.1$ the range is narrower: $\Delta r_{p}=(0.010-0.013) \mathrm{fm}$. Fits $5 \mathrm{~B}$ and $6 \mathrm{~B}$ may be considered the most realistic ones and give

$$
<r^{2}>_{\mathrm{e}-\text { scatt with Coulomb }}^{1 / 2}=(0.880 \pm 0.015) \mathrm{fm} .
$$

Here I have slightly enlarged the errors obtained in both fits 1 . Eq. (14) is now in good agreement with the value (2) deduced from the recent Lamb shift measurements.

Acknowledgements : I remember with gratitude and some sadness many discussions with the late V. Walther and members of his group at Mainz University. His untimely death cut short a very productive and farseeing research. At PSI I would like to thank David Taqqu for a critical reading of the manuscript.

\footnotetext{
${ }^{3}$ The errors given in Table 2 are the "naive" errors obtained from the MINUIT fit program.

${ }^{4}$ Historically there seems to be a tendency to underestimate the (systematic) errors in the proton radius determined from electron scattering and the above value may also be insufficient in this respect. However, a thorough treatment of systematic errors is beyond the scope of the present investigation which focuses on the change of the extracted radius due to Coulomb corrections.
} 


\section{References}

[1] G. G. Simon et al., Nucl. Phys. A 333 (1980) 38

[2] C. N. Hand, D. J. Miller and R. Wilson, Rev. Mod. Phys. 35 (1963) 335

[3] F. Borkowski et al., Nucl. Phys. B 93 (1975) 461

[4] S. Platchkov et al., Nucl. Phys. A 510 (1990) 740

[5] C. Schwob et al., Phys. Rev. Lett. 82 (1999), 4960

[6] K. Melnikov and T. van Ritbergen, preprint hep-ph/9911277

[7] I. Sick and D. Trautmann, Phys. Lett. B 375 (1996) 16

[8] T. Herrmann and R. Rosenfelder, Eur. Phys. J. A 2 (1998) 29

[9] F. Kottmann et al., PSI-proposal R-98-03 (December 1998)

[10] J. L. Friar, Ann. Phys. 98 (1976) 490

[11] L. L. Foldy, K. W. Ford and D. R. Yennie, Phys. Rev. 113 (1959) 1147

[12] L. Andivahis et al., Phys. Rev. D 50 (1994) 5491

[13] B. D. Milbrath et al., Phys. Rev. Lett. 80 (1998) 452;

M. K. Jones et al., preprint nucl-ex/9910005

[14] G. G. Simon, Ch. Schmitt and V. H. Walther, Nucl. Phys. A 364 (1981) 285

[15] F. Borkowski et al., Nucl. Phys. A 222 (1974) 269

[16] W. Reuter, PhD dissertation (1981), report KPH 4/81

[17] J. J. Murphy, Y. M. Shin and D. M. Skopik, Phys. Rev. C 9 (1974) 2125

[18] Ch. Wong, Int. J. Mod. Phys. 3 (1994) 821

[19] F. Borkowski et al., Z. Phys. A 275 (1975) 29 
Table 1: Electron-proton scattering experiment, incident energy, scattering angle, four momentum transfer squared and measured cross section with error (in units of the last digit). $\delta_{\text {norm }}$ denotes the normalization error as given in the references. $\Delta_{\text {mag }}$ is the correction for magnetic scattering, $\Delta_{c}$ the Coulomb correction (see text). The final column gives the electric form factor and its error, again in units of the last digit. Entries which are blank have the same value as in the previous line.

\begin{tabular}{|c|c|c|c|c|c|c|c|c|}
\hline Exp. & $E_{i}[\mathrm{MeV}]$ & $\Theta\left[^{\circ}\right]$ & $Q^{2}\left[\mathrm{fm}^{-2}\right]$ & $\begin{array}{c}\sigma(\delta \sigma) \\
{\left[10^{-4} \mathrm{fm}^{2} / \mathrm{sr}\right]}\end{array}$ & $\delta_{\text {norm }}$ & $\Delta_{\mathrm{mag}}$ & $\Delta_{c}$ & $G_{E}\left(\delta G_{E}\right)$ \\
\hline \multirow[t]{5}{*}{ S1 } & 149.84 & 27.965 & 0.1322 & $60.72(106)$ & $0.0048^{a}$ & 0.0128 & 0.0045 & $0.9770(86)$ \\
\hline & 228.65 & & 0.3049 & $25.47 \quad(27)$ & & 0.0296 & 0.0042 & $0.9634(52)$ \\
\hline & 274.83 & & 0.4380 & $17.25 \quad(18)$ & & 0.0426 & 0.0040 & $0.9504(51)$ \\
\hline & 301.42 & & 0.5252 & $13.95 \quad(13)$ & & 0.0511 & 0.0039 & $0.9356(45)$ \\
\hline & 321.26 & & 0.5952 & $12.10 \quad(10)$ & & 0.0580 & 0.0038 & $0.9272(40)$ \\
\hline \multirow[t]{18}{*}{$\mathrm{S} 2$} & 149.4 & 28 & 0.1317 & $61.96 \quad(72)$ & $0.0056^{b}$ & 0.0128 & 0.0045 & $0.9866(58)$ \\
\hline & & 30 & 0.1504 & $46.19 \quad(47)$ & & 0.0149 & 0.0047 & $0.9797(51)$ \\
\hline & & 35 & 0.2015 & $24.51 \quad(23)$ & & 0.0209 & 0.0052 & $0.9763(47)$ \\
\hline & & 40 & 0.2586 & $13.94 \quad(14)$ & & 0.0283 & 0.0055 & $0.9674(50)$ \\
\hline & & 45 & 0.3208 & $8.43 \quad(8)$ & & 0.0373 & 0.0058 & $0.9582(47)$ \\
\hline & & 50 & 0.3875 & $5.403 \quad(52)$ & & 0.0481 & 0.0061 & $0.9536(47)$ \\
\hline & & 55 & 0.4578 & $3.598 \quad(40)$ & & 0.0612 & 0.0063 & $0.9485(54)$ \\
\hline & & 60 & 0.5310 & $2.428 \quad(25)$ & & 0.0768 & 0.0064 & $0.9344(50)$ \\
\hline & & 65 & 0.6062 & $1.718 \quad(18)$ & & 0.0954 & 0.0066 & $0.9296(51)$ \\
\hline & & 70 & 0.6828 & $1.225 \quad(14)$ & & 0.1176 & 0.0066 & $0.9175(55)$ \\
\hline & 199.5 & 28 & 0.2335 & $33.64 \quad(35)$ & & 0.0227 & 0.0044 & $0.9695(52)$ \\
\hline & & 30 & 0.2663 & $25.31 \quad(26)$ & & 0.0263 & 0.0045 & $0.9670(51)$ \\
\hline & & 35 & 0.3560 & $13.34 \quad(14)$ & & 0.0369 & 0.0049 & $0.9598(52)$ \\
\hline & & 40 & 0.4556 & $7.478 \quad(66)$ & & 0.0499 & 0.0051 & $0.9432(43)$ \\
\hline & & 45 & 0.5637 & $4.493 \quad(41)$ & & 0.0656 & 0.0053 & $0.9300(44)$ \\
\hline & & 50 & 0.6787 & $2.865 \quad(27)$ & & 0.0845 & 0.0054 & $0.9216(45)$ \\
\hline & & 55 & 0.7993 & $1.866 \quad(22)$ & & 0.1072 & 0.0055 & $0.9045(56)$ \\
\hline & & 60 & 0.9239 & $1.283 \quad(17)$ & & 0.1341 & 0.0055 & $0.8970(62)$ \\
\hline \multirow[t]{10}{*}{$\mathrm{R}$} & 100 & 30 & 0.0678 & $104.9 \quad(10.5)$ & $0.011^{c}$ & 0.0067 & 0.0048 & $0.9883(51)$ \\
\hline & & 40 & 0.1172 & $31.71 \quad(25)$ & & 0.0128 & 0.0058 & $0.9772(40)$ \\
\hline & & 50 & 0.1767 & $12.54 \quad(10)$ & & 0.0219 & 0.0066 & $0.9747(40)$ \\
\hline & & 60 & 0.2438 & $5.805 \quad(70)$ & & 0.0351 & 0.0072 & $0.9723(60)$ \\
\hline & & & & $5.751 \quad(46)$ & & & & $0.9677(40)$ \\
\hline & & & & $5.768 \quad(69)$ & & & & $0.9692(59)$ \\
\hline & & 70 & 0.3158 & $2.934 \quad(29)$ & & 0.0542 & 0.0076 & $0.9607(49)$ \\
\hline & & 80 & 0.3901 & $1.627 \quad(16)$ & & 0.0814 & 0.0080 & $0.9567(49)$ \\
\hline & & 90 & 0.4642 & $0.9478(76)$ & & 0.1209 & 0.0082 & $0.9486(41)$ \\
\hline & 300 & 28 & 0.5216 & $14.01 \quad(14)$ & & 0.0508 & 0.0039 & $0.9356(48)$ \\
\hline
\end{tabular}


Table 1 (continued)

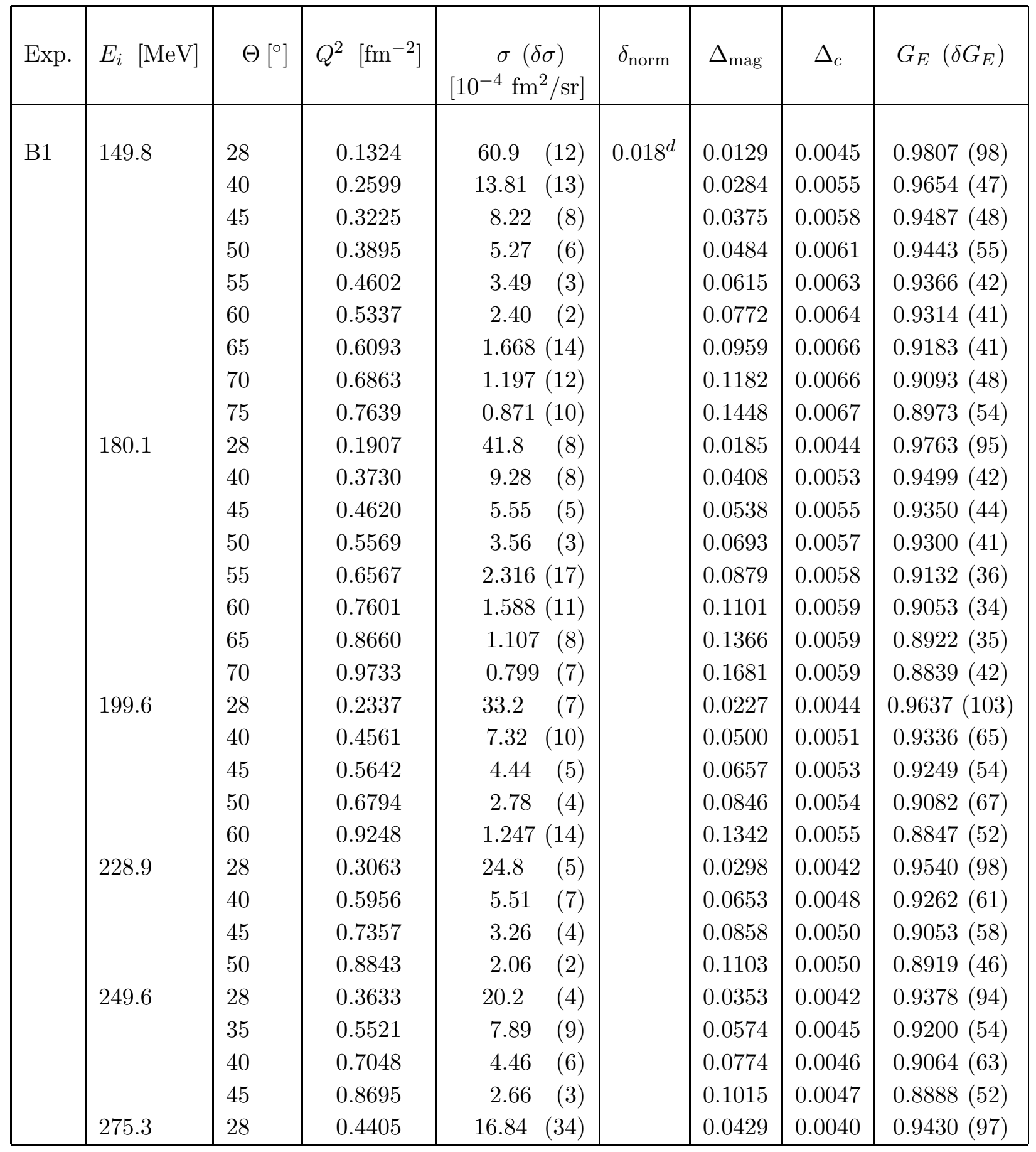


Table 1 (continued)

\begin{tabular}{|c|c|c|c|c|c|c|c|c|}
\hline Exp. & $E_{i}[\mathrm{MeV}]$ & $\Theta\left[^{\circ}\right]$ & $Q^{2}\left[\mathrm{fm}^{-2}\right]$ & $\begin{array}{c}\sigma(\delta \sigma) \\
{\left[10^{-4} \mathrm{fm}^{2} / \mathrm{sr}\right]}\end{array}$ & $\delta_{\text {norm }}$ & $\Delta_{\mathrm{mag}}$ & $\Delta_{c}$ & $G_{E}\left(\delta G_{E}\right)$ \\
\hline \multirow[t]{16}{*}{ B2 } & \multirow[t]{2}{*}{149.8} & 80 & 0.8414 & $0.659 \quad(6)$ & \multirow[t]{16}{*}{$0.018^{d}$} & 0.1766 & 0.0067 & $0.8947(44)$ \\
\hline & & 90 & 0.9939 & $0.386 \quad(3)$ & & 0.2606 & 0.0067 & $0.8786(38)$ \\
\hline & \multirow[t]{4}{*}{228.9} & 28 & 0.3063 & $24.5 \quad(5)$ & & 0.0298 & 0.0042 & $0.9482(98)$ \\
\hline & & 40 & 0.5956 & $5.46 \quad(7)$ & & 0.0653 & 0.0048 & $0.9220(61)$ \\
\hline & & 45 & 0.7357 & $3.23 \quad(4)$ & & 0.0858 & 0.0050 & $0.9011(58)$ \\
\hline & & 50 & 0.8843 & $2.04 \quad(2)$ & & 0.1103 & 0.0050 & $0.8876(46)$ \\
\hline & \multirow[t]{4}{*}{249.6} & 28 & 0.3633 & $20.4 \quad(4)$ & & 0.0353 & 0.0042 & $0.9425(94)$ \\
\hline & & 35 & 0.5521 & $7.97 \quad(9)$ & & 0.0574 & 0.0045 & $0.9246(54)$ \\
\hline & & 40 & 0.7048 & $4.51 \quad(6)$ & & 0.0774 & 0.0046 & $0.9115(63)$ \\
\hline & & 45 & 0.8695 & $2.69 \quad(3)$ & & 0.1015 & 0.0047 & $0.8938(52)$ \\
\hline & \multirow[t]{2}{*}{275.3} & 35 & 0.6685 & $6.43 \quad(7)$ & & 0.0695 & 0.0043 & $0.9136(52)$ \\
\hline & & 40 & 0.8523 & $3.59 \quad(4)$ & & 0.0936 & 0.0044 & $0.8937(52)$ \\
\hline & \multirow[t]{4}{*}{298.5} & 28 & 0.5165 & $13.92 \quad(15)$ & & 0.0503 & 0.0039 & $0.9280(52)$ \\
\hline & & 30 & 0.5881 & 10.34 (11) & & 0.0583 & 0.0040 & $0.9188(51)$ \\
\hline & & 35 & 0.7826 & $5.31 \quad(6)$ & & 0.0814 & 0.0041 & $0.8978(53)$ \\
\hline & & 40 & 0.9966 & $2.96 \quad(3)$ & & 0.1096 & 0.0041 & $0.8768(47)$ \\
\hline \multirow[t]{11}{*}{ M } & 57.3 & 86.61 & 0.1500 & $3.557(36)$ & \multirow[t]{11}{*}{$0.046^{e}$} & 0.0359 & 0.0090 & $0.9768(51)$ \\
\hline & 82.2 & 85.19 & 0.2943 & $1.833(19)$ & & 0.0683 & 0.0085 & $0.9647(52)$ \\
\hline & 82.4 & 85.18 & 0.2957 & 1.815 (11) & & 0.0686 & 0.0085 & $0.9620(31)$ \\
\hline & 89.7 & 84.78 & 0.3456 & $1.555(10)$ & & 0.0795 & 0.0084 & $0.9571(33)$ \\
\hline & 95.6 & 84.45 & 0.3883 & 1.401 & & 0.0887 & 0.0082 & $0.9582(33)$ \\
\hline & 96.4 & 84.41 & 0.3943 & $1.362 \quad(9)$ & & 0.0900 & 0.0082 & $0.9514(34)$ \\
\hline & 102.2 & 84.09 & 0.4384 & $1.227 \quad(8)$ & & 0.0994 & 0.0080 & $0.9474(33)$ \\
\hline & 108.7 & 83.73 & 0.4900 & $1.089 \quad(9)$ & & 0.1103 & 0.0079 & $0.9381(41)$ \\
\hline & 90.4 & 115.33 & 0.5269 & $0.349 \quad(7)$ & & 0.2730 & 0.0089 & $0.9345(98)$ \\
\hline & 118.6 & 93.23 & 0.6733 & $0.552 \quad(5)$ & & 0.1887 & 0.0077 & $0.9189(45)$ \\
\hline & 129.5 & 92.64 & 0.7874 & $0.475 \quad(4)$ & & 0.2177 & 0.0074 & $0.9107(42)$ \\
\hline
\end{tabular}

S1: ref. [迆, Table 1, systematic errors added linearly to statistical cross section errors

S2: ref. [14], Table 1, systematic error of $0.46 \%$ added linearly to statistical cross section errors

R: ref. [16], Tabelle 11, cross section errors from $G_{E}$ errors

B1: ref. [15], Table 1 ; B2: ref. [3], Table 1

M: ref. [17, Table II, cross sections and errors from $G_{E}, \delta G_{E}$ values

${ }^{a}$ caption to Table 1 in S1; ${ }^{b}$ caption to Table 1 in $\mathrm{S} 2 ;{ }^{c}$ p. 92 , line 14 in R; ${ }^{d}$ p. 387, line 4 in S1;

$e$ Table I in M

$\alpha^{-1}=137.036, \hbar c=197.327 \mathrm{MeV}$ fm, $M_{p}=938.272 \mathrm{MeV}, \mu_{p}=2.79285$. 
Table 2: Fit results: A without, B with Coulomb corrections. Errors are in units of the last digit.

\begin{tabular}{|c|c|c|c|c|c|c|}
\hline \multirow[t]{2}{*}{ Fit } & \multicolumn{2}{|c|}{1} & \multicolumn{2}{|c|}{2} & \multicolumn{2}{|c|}{3} \\
\hline & $\mathrm{A}$ & B & A & B & $\mathrm{A}$ & B \\
\hline$\Delta_{\mathrm{mag}}$ & \multicolumn{2}{|c|}{$<0.1$} & \multicolumn{2}{|c|}{$<0.1$} & \multicolumn{2}{|c|}{$<0.15$} \\
\hline$n$ & \multicolumn{2}{|c|}{70} & \multicolumn{2}{|c|}{70} & \multicolumn{2}{|c|}{85} \\
\hline$a_{2}\left[\mathrm{fm}^{4}\right]$ & \multicolumn{2}{|c|}{$0.011(4)$} & \multicolumn{2}{|c|}{$0.014(4)$} & \multicolumn{2}{|c|}{$0.014(4)$} \\
\hline$\Delta_{c}$ & 0 & $\neq 0$ & 0 & $\neq 0$ & 0 & $\neq 0$ \\
\hline$\chi^{2}$ & 22.00 & 20.97 & 22.39 & 21.08 & 26.83 & 24.68 \\
\hline$a_{0}(\mathrm{~S} 1)$ & $1.0002(19)$ & $0.9999(20)$ & $1.0003(19)$ & $1.0000(20)$ & $1.0004(19)$ & $1.0000(19)$ \\
\hline$a_{0}(\mathrm{~S} 2)$ & $1.0014(17)$ & $1.0002(17)$ & $1.0016(17)$ & $1.0004(17)$ & $1.0017(16)$ & $1.0004(16)$ \\
\hline$a_{0}(\mathrm{R})$ & $1.0002(18)$ & $0.9979(18)$ & $1.0004(18)$ & $0.9981(18)$ & $1.0013(17)$ & $0.9988(17)$ \\
\hline$a_{0}(\mathrm{~B} 1)$ & $0.9938(22)$ & $0.9926(22)$ & $0.9940(22)$ & $0.9928(22)$ & $0.9939(21)$ & $0.9926(21)$ \\
\hline$a_{0}(\mathrm{~B} 2)$ & $0.9910(32)$ & $0.9908(32)$ & $0.9910(32)$ & $0.9908(32)$ & $0.9914(29)$ & $0.9909(30)$ \\
\hline$a_{0}(\mathrm{M})$ & $1.0033(20)$ & $1.0002(21)$ & $1.0036(20)$ & $1.0005(21)$ & $1.0036(19)$ & $1.0003(19)$ \\
\hline$r_{p}[\mathrm{fm}]$ & $0.861(12)$ & $0.871(12)$ & $0.867(12)$ & $0.878(12)$ & $0.870(10)$ & $0.878(10)$ \\
\hline
\end{tabular}

Table 2 (continued)

\begin{tabular}{|c|c|c|c|c|}
\hline \multirow{2}{*}{ Fit } & 4 & 5 & \multicolumn{2}{|c|}{6} \\
\hline & $\mathrm{B}$ & B & $\mathrm{A}$ & B \\
\hline$\Delta_{\mathrm{mag}}$ & $<0.1$ & $<0.1$ & \multicolumn{2}{|c|}{$<0.1$} \\
\hline$n$ & $54^{a}$ & $63^{b}$ & \multicolumn{2}{|c|}{70} \\
\hline$a_{2}\left[\mathrm{fm}^{4}\right]$ & $0.014(4)$ & $0.014(4)$ & \multicolumn{2}{|c|}{$0.014(4)$} \\
\hline$\Delta_{c}$ & $\neq 0$ & $\neq 0$ & 0 & $\neq 0$ \\
\hline$\chi^{2}$ & 14.29 & 19.52 & $9.96^{c}$ & $9.29^{c}$ \\
\hline$a_{0}(\mathrm{~S} 1)$ & $1.0007(20) \quad 1.0004(20)$ & $1.0004(19) \quad 1.0001(20)$ & $1.0003(20)$ & $1.0002(20)$ \\
\hline$a_{0}(\mathrm{~S} 2)$ & $1.0022(17) \quad 1.0010(17)$ & $1.0018(17) \quad 1.0006(17)$ & $1.0016(17)$ & $1.0006(18)$ \\
\hline$a_{0}(\mathrm{R})$ & - & $1.0006(18) \quad 0.9983(18)$ & $1.0004(29)$ & $0.9988(29)$ \\
\hline$a_{0}(\mathrm{~B} 1)$ & $0.9950(23) \quad 0.9938(23)$ & $0.9943(22) \quad 0.9932(22)$ & $0.9944(31)$ & $0.9936(32)$ \\
\hline$a_{0}(\mathrm{~B} 2)$ & $0.9922(33) \quad 0.9921(33)$ & $0.9914(32) \quad 0.9913(32)$ & $0.9923(45)$ & $0.9926(45)$ \\
\hline$a_{0}(\mathrm{M})$ & - & - & $1.0037(33)$ & $1.0009(34)$ \\
\hline$r_{p}[\mathrm{fm}]$ & $0.875(13) \quad 0.885(13)$ & $0.870(12) \quad 0.880(12)$ & $0.868(14)$ & $0.881(14)$ \\
\hline
\end{tabular}

${ }^{a}$ only S1, S2, B1, B2 data sets; $\quad{ }^{b}$ only S1, S2, R, B1, B2 data sets;

${ }^{c}$ errors in data sets R, B1, B2, M enlarged by a factor of 2 